\title{
BMJ Open Cryoablation for advanced non-small cell lung cancer: a protocol for a systematic review
}

\author{
Hua Duan (D) , ${ }^{1}$ Shu-Yue Zheng (D) , ${ }^{1}$ Chufan Luo, ${ }^{1}$ Xueni Fang, ${ }^{2}$ Dan Wang, ${ }^{2}$ \\ Haoyue Pang, ${ }^{1}$ Man Wang, ${ }^{1}$ Yu Chen, ${ }^{1}$ Tian Zhou, ${ }^{2}$ Quanwang Li, ${ }^{2}$ Kaiwen $\mathrm{Hu}^{2}$
}

To cite: Duan H, Zheng S-Y, Luo C, et al. Cryoablation for advanced non-small cell lung cancer: a protocol for a systematic review. BMJ Open 2020;10:e033460. doi:10.1136/ bmjopen-2019-033460

- Prepublication history and additional material for this paper are available online. To view these files, please visit the journal online (http://dx.doi. org/10.1136/bmjopen-2019033460).

Received 11 August 2019 Revised 02 April 2020 Accepted 07 April 2020
Check for updates

(C) Author(s) (or their employer(s)) 2020. Re-use permitted under CC BY-NC. No commercial re-use. See rights and permissions. Published by BMJ.

${ }^{1}$ Graduate School, Beijing University of Chinese Medicine, Beijing, China

${ }^{2}$ Oncology Department, Dongfang Hospital, Beijing University of Chinese Medicine, Beijing, China

Correspondence to

Dr Kaiwen $\mathrm{Hu}$;

kaiwenh@163.com

\section{ABSTRACT}

Introduction National Comprehensive Cancer Network has recommended cryoablation to replace the resection in the treatment of medically operable non-small cell lung cancer (NSCLC). Cryoablation also has been used for the advanced NSCLC in randomised controlled trials. However, they have not been systematically reviewed. Here, we provide a protocol to evaluate the effectiveness and safety of cryoablation in the treatment of advanced NSCLC. Methods and analyses We will search PubMed, Embase, the Cochrane Library, Chinese Biomedical Database, China National Knowledge Infrastructure, Wanfang Database and Chinese Scientific Journal Database without language restrictions from inception until 1 February 2020. Trial registers (International Clinical Trials Registry platform, the US National Institutes of Health Ongoing Trials Register and the ISRCTN registry) and reference lists of retrieved articles will also be searched. Two reviewers will independently extract data on participants, interventions, comparisons, outcomes and assess the methodological quality by the Cochrane risk of bias tool. The strength of evidences will be evaluated according to the Grading of Recommendations Assessment, Development and Evaluation approach. Review Manager V.5.3 software will be used for data analyses. Meta-analyses will be performed if the data are sufficiently homogeneous. The primary outcomes will be objective response rate and overall survival. The secondary outcomes will be adverse effects, health-related quality of life, changes of immune indicators and surrogate outcomes (disease control rate, progression-free survival and survival rate).

Ethics and dissemination Ethics approval is not required, as this study will not involve patients. The results of this study will be submitted to a peer-reviewed journal for publication, to inform both clinical practice and further research. PROSPERO registration number CRD42019138660.

\section{INTRODUCTION}

\section{Description of the condition}

The Global Cancer Statistics from 2018 reveals that lung cancer is the most frequent cancer in both men and women and is the leading cause of cancer-related mortalities. Non-small cell lung cancer (NSCLC) is the most common type of lunch cancer which accounts for $\sim 86 \%$ of cases. ${ }^{2}$ About $75 \%$ of patients with NSCLC were found to

\section{Strengths and limitations of this study}

This will be the first Preferred Reporting Items for Systematic Reviewsand Meta-Analyses compliant systematic review to assess the effectiveness and safety of cryoablation for advanced non-small cell lung cancer.

- Besides the Cochrane risk of bias tool, the Grading of Recommendations Assessment, Development and Evaluation approach will be used to evaluate the strength of evidence.

- Selection, data extraction and bias assessment will be performed by two independent reviewers.

- A limitation of this meta-analysis is that we do not limit the type of cryoablation or chemotherapy, the risk of heterogeneity will occur inevitably.

be at advanced stages at the time of diagnosis where surgery was not a suitable therapeutic option. Although targeted therapy and immunotherapy enhanced the survival benefit of patients with lung cancer when compared with chemoradiotherapy, only patients harbouring sensitive mutations ${ }^{3}$ or high expression levels of immune checkpoint proteins ${ }^{4}$ benefit from these two therapies. Chemotherapy and radiotherapy are still the two main therapies used for patients with advanced-stage lung cancer. For patients receiving platinum-based two-drug regimens as first-line therapies, the median overall survival (OS) is $8-12$ months and the 1-year survival rate is $\sim 40 \% .^{5}$ Adverse events (AEs) of these therapies including radiation pneumonitis, myelosuppression, nausea and vomiting are common in patients.

\section{Description of the intervention}

A prostate tumour was the first cancer treated with cryoablation in the 1970s. Liquid nitrogen, supplied by Cryomedical Science (CMS, USA), was the most common freezing medium used at that time. In 1998, a new device for ultra-low temperature interventional cryoablation, Endocare CryoCare Surgical System, was approved 
by the Food and Drug Administration (FDA). Argon mixed with helium became the new preferred freezing medium. Two years later, a similar device, Galil Medical, generated in Israel was also approved by the FDA. After the generation of these novel devices, cryoablation was more commonly used to treat malignant tumours, including lung cancer. Presently, cryoablation has been recommended to be used in the treatment of medically operable NSCLC when resection is not suitable. ${ }^{6}$ Some patients with poor general health or poor respiratory function also choose cryoablation over chemoradiotherapy. Compared with traditional surgery, cryoablation results in lower trauma, mild adverse reactions, shorter hospital stays and quicker recovery. Compared with chemoradiotherapy, cryoablation focuses on the tumour and minimises damage to surrounding normal tissue.

A phase II multicentre, prospective, single-arm study revealed that the local tumour control rates after receiving Endocare cryoablation were $96.6 \%$ and $94.2 \%$ at 6 and 12 months, respectively. The 1-year OS rate was $97.5 \%$ with no changes in patient quality of life. ${ }^{7}$ Another study that included 46 patients with peripheral NSCLC suggested that average tumour CT values dropped from $32 \pm 10 \mathrm{HU}$ to $-21 \pm 8 \mathrm{HU}$ after Galil Medical cryoablation. Moreover, the complete and partial response rates were $83.7 \%$ and $16.3 \%$, respectively. ${ }^{8}$ Therefore, cryoablation brought survival benefits with tolerable toxicities for patients with both primary and metastatic lung cancers. However, the role of cryoablation in advanced NSCLC is still unclear.

\section{How the intervention might work}

As a local physical therapy, cryoablation exerts antineoplastic actions through multiple aspects. Argon-helium cryoablation, including Endocare and the Galil Medical cryosystem, is the most mature cryoablation strategy used in clinical practices. The Joule-Thomson theory is the essential principle behind cryoablation. By using highpressure argon, the target tumour is cooled to $-140^{\circ} \mathrm{C}$ and then rapidly heated from $+20^{\circ} \mathrm{C}$ to $+40^{\circ} \mathrm{C}$ with helium. ${ }^{6}$ The CMS cryosystem, which uses liquid nitrogen, could cool the tumour to $-196^{\circ} \mathrm{C}$.

Freeze-thaw cycles not only induce cell death by membrane disruption and microvascular thrombosis ${ }^{9-11}$ but also lead to protein denaturation and cellular dehydration due to the presence of extremely low temperatures. ${ }^{10}$ Combined with chemotherapy, cryoablation can inhibit tumour growth compared with single chemotherapy as was shown in mice with severe combined immune deficiency (SCID). ${ }^{12}$ In addition, many experimental studies since the late 1960s have documented the immunological effects of cryoablation. ${ }^{13}{ }^{14}$ Frozen lung cancer cells could release tumour antigens and elicit antitumour immune responses in mouse models. ${ }^{15}$

\section{Why it is important to do this review}

There are three systematic reviews discussing cryoablation for advanced NSCLC combined with chemotherapy, radiotherapy or chemoradiotherapy. However, all reviews are written in in Chinese and consist of different methodologies, and all these reviews are not adequate in respect to systematic reviewing according to the Preferred Reporting Items for Systematic Reviews and Meta-Analyses (PRISMA) statement. ${ }^{16}$ One review ${ }^{17}$ included four studies (published in 2003-2007) that chose a single cryoablation method as the control group. Another review ${ }^{18}$ included five studies (published in 2004-2007) where the control groups included both cryoablation and chemoradiotherapy. Thus, the study design was not completely similar to this protocol. The latest review ${ }^{19}$ included 16 studies between 2003 and 2014 where 10 of these studies were not randomised controlled trials (RCTs) but simple controlled trials. However, as the author did not separately analyse these studies, the results were not rigorous. Based on these shortcomings, the effect of cryoablation for advanced NSCLC has not been fully assessed. Therefore, a comprehensive, up-to-date and PRISMA-compliant systematic review of RCTs is necessary to properly evaluate the effectiveness and safety of cryoablation for advanced NSCLC. The Grading of Recommendations Assessment, Development and Evaluation (GRADE) is used to assess the strength of the evidence.

\section{OBJECTIVES}

This systematic review aims to examine the effectiveness and safety of cryoablation for the patients with advanced NSCLC.

\section{METHODS \\ Criteria for considering studies for this review \\ Types of studies}

Only RCTs will be eligible for inclusion irrespective of language. Case reports, case series, commentaries and quasi-RCTs will be excluded.

\section{Types of participants}

Participants (18 years and older) with advanced NSCLC (stage III-IV) diagnosed by histology or pathology will be included. There will be no restrictions on gender or ethnicity.

\section{Types of interventions}

Studies assessing cryoablation for patients with advanced NSCLC regardless of procedure will be included. Control intervention should be chemotherapy, radiotherapy or chemoradiotherapy regardless of dosage and type. The control group should have the same treatment besides cryoablation compared with the experimental group.

\section{Types of outcome measures}

\section{Primary outcomes}

Primary outcomes will include: (a) objective response rate (ORR), measured by Response Evaluation Criteria in Solid Tumors or WHO criteria; (b) OS. 
Secondary outcomes

Secondary outcomes will include: (a) AEs; (b) healthrelated quality of life (measured by a validated tool such as the Performance Status, Karnofsky Performance Score, Eastern Cooperative Oncology Group, Quality of Life Questionnaire-Core and so on); (c) disease control rate (DCR), measured by Response Evaluation Criteria in Solid Tumors or WHO criteria; (d) progression-free survival; (e) survival rate; (f) changes of immune indicators (specifically the activity of natural killer (NK) cells and the T-lymphocyte subset).

\section{Search methods for identification of studies \\ Electronic searches}

The following databases will be searched from their inception to 1 February 2020: PubMed, Embase, Cochrane Central Register of Controlled Trials, Chinese Biomedical Database, China National Knowledge Infrastructure, Wanfang Database and Chinese Scientific Journal Database.

\section{Searching other resources}

Clinical trials registry platforms will be searched, including the International Clinical Trials Registry platform (http://www.who.int/ictrp/network/primary/ en/), the US National Institutes of Health Ongoing Trials Register (http://clinicaltrials.gov/) and the ISRCTN registry (http://www.controlled-trials.com/). We will also screen the reference lists of retrieved articles to identify missing eligible studies.

\section{Search strategies}

Search strategies in English electronic databases will be listed in the online supplementary appendix A and will be adapted for other resources with appropriate terms. No language restriction will be applied.

\section{Data collection and analysis}

\section{Selection of studies}

Two reviewers (HD, S-YZ) will independently screen the titles and abstracts of these studies. Potentially eligible studies will be confirmed by evaluating the full text. Any uncertainty or disagreements will be resolved by a third investigator (TZ). Details of the study screening process will be shown as in figure 1 .

\section{Data extraction and management}

Two reviewers (HD, S-YZ) will independently extract data from the selected studies with a predesigned data form. Disagreements will be resolved by discussion or consulting a third reviewer (TZ). We will seek missing data by contacting the original authors whenever possible. The following information was extracted:

a. Studies (publication years, number of authors, the first author, study design, sample size, country, follow-up and quality of study).

b. Participants (age, gender, race, stage of NSCLC and loss to follow-up).

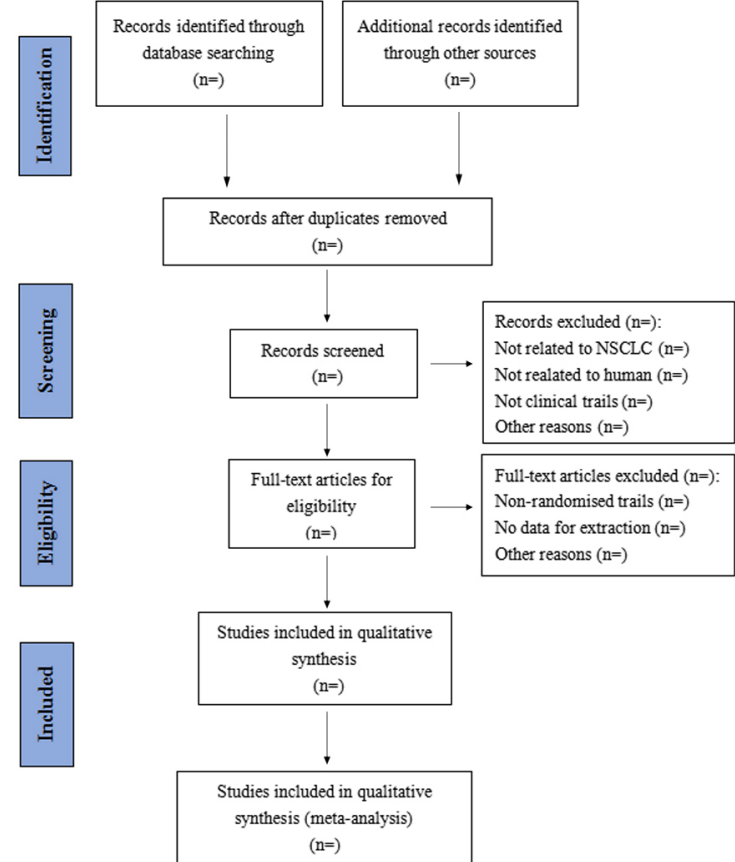

Figure 1 Flow diagram of study search and identification. NSCLC, non-small cell lung cancer.

c. Interventions (dosage, duration, cryoablation, chemotherapy and radiotherapy).

d. Outcome measures, results and AEs.

\section{Assessment of risk of bias in included studies}

Two reviewers (HD, S-YZ) will independently evaluate the risk of bias for each included study using the Cochrane Collaboration's risk of bias tool, consisting of the following items: random sequence generation, allocation concealment, blinding of participants and personnel, blinding of outcome assessment, incomplete outcome data, selective reporting and other bias. We will judge each item as low, high or unclear risk of bias. Any disagreements will be arbitrated by a third reviewer (TZ).

\section{Measures of treatment effect}

The effect size of the included studies was assessed with different measures based on the type of data. Continuous data were expressed as the weighted mean difference or standardised mean difference. Dichotomous data were expressed as the relative risk (RR) (eg, ORR). Survival data were assessed with the HRs and 95\% CIs. If we cannot directly extract from the texts, we used Engauge Digitizer (V.4.1) to extract HRs and 95\% CIs by computing the Kaplan-Meier graph. A 95\% CI was used for all data analyses.

\section{Dealing with missing data}

In case of missing data, we will contact the authors. If we failed to obtain the missing data, those dates will be excluded from the analysis.

\section{Assessment of heterogeneity}

Cochran's Q-test and I ${ }^{2}$ statistics were used to evaluate the heterogeneity of the included studies. A Q-test with 
p $>0.10$ and an $\mathrm{I}^{2}$ of no more than $50 \%$ indicated that statistical homogeneity was acceptable. Otherwise, we analysed the potential cause of heterogeneity, which included the methodological characteristics of the study and the biological characteristics of the participants. Subgroup analysis and sensitivity analysis were used to account for heterogeneity.

\section{Assessment of reporting biases}

Reporting biases or small-study effects were checked by funnel plots generated from data of more than eight studies.

\section{Data synthesis}

Statistical analyses were performed using Review Manager V.5.3 software (The Cochrane Collaboration). The selected clinical studies were descriptively analysed, and summary statistics were presented. If studies were sufficiently homogeneous, data across studies were pooled for meta-analysis. If the Q-test indicated $\mathrm{p}>0.10$, we used a fixed-effect model. Otherwise, we used a random-effect model, and the potential cause of heterogeneity was interpreted by subgroup analyses and sensitivity analyses. On the other hand, if a meta-analysis was not feasible because of significant statistical heterogeneity, we conducted a descriptive analysis.

\section{Subgroup analysis and investigation of heterogeneity}

If the data are available and sufficient, we will conduct a subgroup analysis to determine the potential cause of heterogeneity. The different stages, chemotherapeutic and comparative interventions and so on will be divided into subgroups for analysis according to the actual conditions.

\section{Sensitivity analysis}

We also conducted sensitivity analysis to verify the study's conclusions. We removed each study one by one and reanalysed the remaining studies. The before and after results were compared to determine the stability of the integrative results.

\section{Grading the quality of evidence}

The quality of evidence for outcomes in the review was assessed according to the GRADE. This classifies the evidence into four grades: high, moderate, low and very low quality. ${ }^{20}$

\section{Patient and public involvement}

Patients and/or the public were not involved in the design, or conduct, or reporting, or dissemination plans of this research.

\section{ETHICS AND DISSEMINATION}

Ethics approval is not required, as this study will not involve patients. The results of this study will be submitted to a peer-reviewed journal for publication to inform both clinical practice and further research.
DISCUSSION

Worldwide, lung cancer is the leading cause of cancerrelated deaths, with an incidence of about 2.5 million, leading to 1.6 million deaths each year. Data from China suggested that there will be 733300 new cases and 610200 more deaths from lung cancer, respectively. ${ }^{21}$ Most patients with unresectable lung cancer only derive limited benefits from traditional radiotherapy and chemotherapy due to the absence of PD-L1 expression or mutations in critical oncogenes. For patients with advanced NSCLC receiving platinum-based chemotherapy, the median OS was only $8-12$ months $^{5}$ and the RR was $\sim 20 \%-30 \% .^{22} 23$ Combined with other therapies, this could be a meaningful research direction.

By directly freezing deep lesions, cryoablation results in protein denaturation, cellular dehydration and induces cell death through membrane disruption and microvascular thrombosis. Various experimental and clinical studies have shown the antineoplastic and immunological effects of cryoablation. The method of localised targeting leads to less trauma, mild adverse reactions and rapid recovery. Besides combining with chemoradiotherapy, cryoablation in addition to allogenic NK cell immunotherapy $^{24}$ could significantly increase the RR and DCR compared with cryoablation alone. Another study found that cryoablation combined with traditional Chinese medicine could be used as the main therapy for the elderly or patients with advanced-stage lung cancer in China. ${ }^{25}$ Therefore, cryoablation plays a unique role in advanced lung cancer cases.

There may be various synergistic mechanisms ${ }^{26}$ between cryoablation and chemotherapy including: (a) cryoablation damages endothelial cells of capillaries and increases the permeability of blood vessels, which facilitates drug penetration; (b) chemotherapy reduces tumour volume, which is conducive to completely freezing and killing the tumour; and (c) immune enhancement caused by cryoablation is beneficial to reduce immune function decline caused by chemotherapy and can reduce AEs of chemotherapy. These potential mechanisms provide the theoretical basis for combining cryoablation and chemotherapy.

This systematic review will be the first to evaluate the effectiveness and safety of cryoablation for advanced NSCLC using the PRISMA and GRADE approaches. Although an extensive and unbiased search will be conducted, it is difficult to obtain raw data for RCTs and unpublished RCTs that reveal negative results will not be included. However, this review will still provide objective evidence for the use of cryoablation in the treatment of advanced NSCLC.

Contributors HD and S-YZ are joint first authors. KH conceived and designed the study. HD,CL developed the search strategy. XF, DW,and HP provided methodological perspectives. $H D$ and $S-Y Z$ drafted and refined the study protocol with contributions from all coauthors $(\mathrm{HD}, \mathrm{S}-\mathrm{YZ}, \mathrm{CL}, \mathrm{XF}, \mathrm{DW}, \mathrm{HP}, \mathrm{MW}, \mathrm{YC}, \mathrm{TZ}, \mathrm{QL}$, $\mathrm{KH}) . \mathrm{HD}, \mathrm{S}-\mathrm{YZ}, \mathrm{MW}, \mathrm{YC}$ will search articles, conduct study selection, perform data extraction, assess the quality and conduct data analyses. QL and TZ will verify data extraction and data analyses. All authors read and approved the final manuscript. 
Funding This work is supported by the National Key R\&D Program of China (No 2018YFC1705102) and Capital's Funds for Health Improvement and Research (No CFH 2018-1-4201).

\section{Competing interests None declared.}

Patient consent for publication Not required.

Provenance and peer review Not commissioned; externally peer reviewed.

Open access This is an open access article distributed in accordance with the Creative Commons Attribution Non Commercial (CC BY-NC 4.0) license, which permits others to distribute, remix, adapt, build upon this work non-commercially, and license their derivative works on different terms, provided the original work is properly cited, appropriate credit is given, any changes made indicated, and the use is non-commercial. See: http://creativecommons.org/licenses/by-nc/4.0/.

\section{ORCID iDs}

Hua Duan http://orcid.org/0000-0002-9768-2428

Shu-Yue Zheng http://orcid.org/0000-0002-4805-0483

\section{REFERENCES}

1 Bray F, Ferlay J, Soerjomataram I, et al. Global cancer statistics 2018: GLOBOCAN estimates of incidence and mortality worldwide for 36 cancers in 185 countries. CA Cancer J Clin 2018;68:394-424.

2 Cheng I, Le GM, Noone A-M, et al. Lung cancer incidence trends by histology type among Asian American, native Hawaiian, and Pacific Islander populations in the United States, 1990-2010. Cancer Epidemiol Biomarkers Prev 2014;23:2250-65.

3 Wu Y-L, Zhou C, Liam C-K, et al. First-Line erlotinib versus gemcitabine/cisplatin in patients with advanced EGFR mutationpositive non-small-cell lung cancer: analyses from the phase III, randomized, open-label, ensure study. Ann Oncol 2015;26:1883-9.

4 Liu J, Zhong Y, Peng S, et al. Efficacy and safety of PD1/PDL1 blockades versus docetaxel in patients with pretreated advanced non-small-cell lung cancer: a meta-analysis. Onco Targets Ther 2018;11:8623-32.

5 Reck M, Rabe KF. Precision diagnosis and treatment for advanced non-small-cell lung cancer. N Engl J Med 2017;377:849-61.

6 Ye X, Fan W, Wang H, et al. Expert consensus workshop report: guidelines for thermal ablation of primary and metastatic lung tumors (2018 edition). J Cancer Res Ther 2018;14:730-43.

7 de Baere T, Tselikas L, Woodrum D, et al. Evaluating Cryoablation of Metastatic Lung Tumors in Patients--Safety and Efficacy: The ECLIPSE Trial--Interim Analysis at 1 Year. J Thorac Oncol 2015;10:1468-74.

8 Zhang X, Tian J, Zhao L, et al. Ct-Guided conformal cryoablation for peripheral NSCLC: initial experience. Eur J Radiol 2012;81:3354-62.

9 de Freitas RMC, Andrade CS, Caldas JGMP, et al. Image-Guided cryoablation of the spine in a swine model: clinical, radiological, and pathological findings with light and electron microscopy. Cardiovasc Intervent Radiol 2015;38:1261-70.
10 Gage AA, Baust J. Mechanisms of tissue injury in cryosurgery. Cryobiology 1998;37:171-86.

11 Guan L, Wang J, Gao K, et al. Percutaneous intraductal radiofrequency ablation combined with biliary stent placement for malignant biliary obstruction: a case report and review of the literature. J Cancer Res Ther 2016;12:C221-4.

12 Forest V, Peoc'h M, Campos L, et al. Benefit of a combined treatment of cryotherapy and chemotherapy on tumour growth and late cryo-induced angiogenesis in a non-small-cell lung cancer model. Lung Cancer 2006;54:79-86.

13 Shulman S, Bronson P, Riera C, et al. Studies in cryo-immumology. 3. the immunoglobulin nature of the antibody response. Immunology 1968;14:541-51.

14 Yantorno C, Soanes WA, Gonder MJ, et al. Studies in cryoimmunology. I. the production of antibodies to urogenital tissue in consequence of freezing treatment. Immunology 1967;12:395-410.

15 Takahashi Y, Izumi Y, Matsutani N, et al. Optimized magnitude of cryosurgery facilitating anti-tumor immunoreaction in a mouse model of Lewis lung cancer. Cancer Immunol Immunother 2016;65:973-82.

16 Moher D, Shamseer L, Clarke M, et al. Preferred reporting items for systematic review and meta-analysis protocols (PRISMA-P) 2015 statement. Syst Rev 2015;4:1.

17 Fei XS, Song Y, Shi Y. Meta-Analysis of cryotherapy combined with chemotherapy for advanced non-small cell lung cancer. $J$ Med Postgrad 2009;22:165-8.

18 XF D, Han BS, TZ L. Effect of argon-helium cryoablation on intermediate and advanced non-small cell lung cancer: a metaanalysis. J Chinese PLA Postgrad Med Sch 2010;31:714-7.

19 Wei Y, Li Z, DH M, et al. Argon helium knife combined with radiotherapy or chemotherapy in treatment of advanced NSCLC: a meta analysis. J Pract Oncol 2015;30:133-9.

20 Balshem H, Helfand M, Schünemann HJ, et al. Grade guidelines: 3. rating the quality of evidence. J Clin Epidemiol 2011;64:401-6.

21 Chen W, Zheng R, Baade PD, et al. Cancer statistics in China, 2015. CA Cancer J Clin 2016;66:115-32.

22 Rosell R, Carcereny E, Gervais R, et al. Erlotinib versus standard chemotherapy as first-line treatment for European patients with advanced EGFR mutation-positive non-small-cell lung cancer (EURTAC): a multicentre, open-label, randomised phase 3 trial. Lancet Oncol 2012;13:239-46.

23 Zhou C, Wu Y-L, Chen G, et al. Erlotinib versus chemotherapy as first-line treatment for patients with advanced EGFR mutationpositive non-small-cell lung cancer (optimal, CTONG-0802): a multicentre, open-label, randomised, phase 3 study. Lancet Oncol 2011;12:735-42.

24 Lin M, Liang S-Z, Wang X-H, et al. Clinical efficacy of percutaneous cryoablation combined with allogenic NK cell immunotherapy for advanced non-small cell lung cancer. Immunol Res 2017;65:880-7.

25 Gao L, Li Q, Jiang M, et al. Combined therapy of percutaneous cryoablation and traditional Chinese medicine can be a promising strategy for elderly or advanced lung cancer patients based on a retrospective clinical study. Cryobiology 2014;69:174-7.

26 Li P, Li W, Sun Z, et al. Status and prospect of argon-helium cryosurgery system for the treatment of gliomas. Chin J Clin Oncol 2014;41:345-8. 